\title{
MENSAGEM DO DIRECTOR DA FACULDADE DE DIREITO DE SÃO PAULO
}

\section{A FACULDADE DE DIREITO DE COIMBRA}

Por occasião da viagem da Embaixada Academica a Portugal, foi entregue a seguinte mensagem, assignada pelo professor Francisco Morato, director da Faculdade de Direito da Universidade de São Paulo, ao director da Faculdade de Direito de Coimbra:

“São Paulo, 31 de maio de 1937.

Exmo. Senhor,

São portadores desta mensagem dezenove alumnos de nossa Faculdade, que partiram hontem pelo BAGE' do Lloyd Brasileiro, chefiados pelo quint'annista Asdrubal de Moraes Andrade e acompanhados de nosso secretario Flavio Mendes.

Vão em missão official e academica levar á Universidade e á Faculdade de Direito de Coimbra, ao Exmo. Sr. Reitor e a V Exa., aos srs. professores e academicos desse centro de inteilectualidade portugueza, a expressão fraternal da sympathia e solidariedade da Faculdade de Direito de São Paulo, nossas, de seus professores $e$ de seu corpo discente. 
Esperamos que sejam acolhidos com a costumada gentileza e que sua visita marque o inicio entre as nossas Escolas de um mais assiduo entretenimento cultural. Quanto mais recúa no tempo a data gloriosa de sua fundação, mais recrescem e apertam na Faculdade de Direito de São Paulo os affectos e lembranças agradecidas que a jungem á sua mãe espiritual de Coimbra. Queira V Exa. acceitar os protestos de nossos sentimentos de respeitosa sympathia e admiração.

Ao Exmo. Sr. Director da

Faculdade de Direito de Coimbra.

(a) Dr. FRANCISCO MORATO"

\section{O HYMNO ACADEMICO}

Em carta dirigida á Chefia Technica da Bibliotheca, assim se referiu o professor Manoel Pacheco Prates, lente jubilado da Faculdade de Direito e actualmente morador em Porto Alegre, Rio Grande do Sul, sobre a chronica de reminiscencias "O Hymno Academico", de autoria do professor Francisco Morato e inserta no fasciculo anterior :

"Exmo. Sr.

António Constantino

Dignissimo Chefe Technico da Bibliotheca

Affectuosas saudações.

Recebi e penhorado agradeço o numero da "Revista da Faculdade de Direito", volume XXXIII, fasciculo I, de janeiro-abril do corrente anno.

Peço venia para dizer que, além dos importantes trabalhos da "Revista", me causou emocionante impressão o bello artigo do eminente collega e querido amigo dr. Morato - sobre o hymno e poesia, e tambem as evocativas gravuras da nossa querida Faculdade.

$O$ velho filho muito grato da Faculdade

(a) M. Pacheco Prates" 


\section{"REVISTA DA FACULDADE DE DIREITO" Opinióes do estrangeiro}

A respeito da "Revista da Faculdade de Direito", recebeu a Chefia Technica da Bibliotheca as cartas abaixo que demonstram o interesse com que está sendo recebida esta publicação nos paizes estrangeiros.

Do professor Ludwik Ehrlich da Universidade Jean Casimir, de Lwów, Polonia:

"Lwów (Pologne), 15-XII-1936.

Monsieur le Chef Technique de la Bibliothèque de la Faculté de Droit de l'Université de São Paulo (Brésil)

Monsieur le Directeur

Je m'empresse de vous remercier chaleuresement de votre envoi des numéros déjà parus de la "Revista" de la Faculté de Droit de l'Université de São Paulo.

Nous vous envoyons par retour du courrier nos publications en Français. Je me permets en outre d'y joindre quelques uns de mes travaux parus en langue anglaise. Nous nous mettons actuellement à publier en Français d'une façon plus active et nous ne tarderons pas à vous envoyer des exemplaires de nos publications des qu'elles paraîtront.

Je vous prie de bien vouloir nous faire parvenir désormais les numéros de la "Revista" de votre Faculté. Elle me paraît en effet extrêmement importante.

Veuillez agréer, Monsieur le Directeur, mes salutations distinguées.

(a) Ludwik Ehrlich"

Do dr. Rodolpho Aladár Métall, redactor da "Revue Internationale de la Théorie du Droit", de Genève, Suisse:

“Genève le 15 avril 1937.

M. António Constantino, Chef Tecnique de la Bibliothèque de la Faculté de Droit de l'Université de São Paulo (Brésil).

Je viens de recevoir votre aimable lettre du 8 mars 1937 ainsi que les fascicules I et II de l'année 1936 de votre excellente "Revista da Faculdade de Direito de São Paulo", et je vous en remercie très vivement.

C'est avec le plus vif intérêt que j'ai pris connaissance du contenu si riche et si varié de la "Revista" Je me ferai un devoir d'attirer l'attention des lecteurs de notre Revue internationale sur les articles publiés dans les deux fascicules de votre "Revista" que vous avez 
bien voulu mettre à ma disposition. Les justificatifs vous seront envoyés, par notre éditeur, des la publication de notre prochain numéro.

Je serais heureux si je pouvais continuer à recevoir votre remarquable "Revista" afin que j'en puisse régulièrement rendre compte dans notre rubrique "Revue des revues"

En attendant le plaisir de vous lire, je vous prie de croire, Monsieur, à l'expression de mes sentiments les meilleurs.

(a) Dr. Métall"

\section{BIBLIOTHECA DA FACULDADE DE DIREITO}

\section{A frequencia no primeiro semestre de $\mathbf{1 9 3 7}$}

Foi consideravel o augmento da frequencia de estranhos e estudantes, na Bibliotheca, durante o primeiro semestre do corrente anno, conforme se verifica dos dados seguintes:

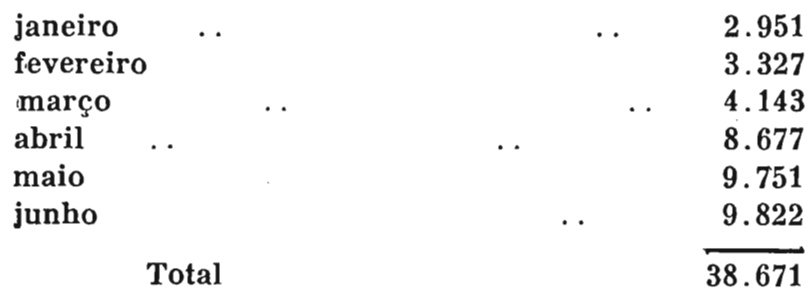

Confrontando-se com a frequencia dos annos anteriores, e mesmo com a de 1936 que excedeu todas as espectativas, nota-se que no decorrer de 1937 o numero de frequentadores será maior. 'E' facil a demonstração:

\begin{tabular}{|c|c|c|c|}
\hline 1932 & & 3.432 & leitores \\
\hline 1933 & & 9.399 & $"$ \\
\hline 1934 & . & 16.240 & " \\
\hline 1935 & & 30.980 & $"$ \\
\hline 1936 & . & 50.419 & $"$ \\
\hline 1937 (1.0 semestre) & & 38.671 & " \\
\hline
\end{tabular}

\section{Impressões de visitantes}

Do livro de visitas constam impressões de personalidades notaveis sobre a actual organização da Bibliotheca da Faculdade de Direito, impressões que attestam a importancia e o desenvolvimento daquelle instituto. 
Na impossibilidade de transcrevel-as todas, reproduzimos as dos ultimos visitantes.

Do professor Clovis Bevilaqua, eminente jurisconsulto e professor honorario da Faculdade:

"Acabamos de percorrer a Bibliotheca da Faculdade de Direito da Universidade de São Paulo. E' admiravel a organização dada pelo distincto collega, encarregado deste serviço. Difficilmente poder-se-á encontrar uma organização de tal modo completa e intelligentemente feita por mão altamente competente.

Sahimos não digo satisfeitos, mas admirados, o gue é muito mais".

Do professor Magalhães Drummond, lente cathedratico da Faculdade de Direito da Universidade de Minas Geraes, e do desembargador Polycarpo de Azevedo Junior, da Côrte de Appellação de São Paulo:

“Excellente a impressão que experimenta quem visite a Bibliotheca da Faculdade de Direito de São Paulo. Vista de longe, através de alheias impressões, imaginase algo de muito grande; vista de perto, verifica-se tratar-se de obra muito maior, inexcedivel mesmo. $E$ ' incalculavel o valor da contribuição que esta Bibliotheca, com a sua sábia organização, traz para a cultura brasileira"

Do dr. A. Bento de Faria, ministro da Côrte Suprema:

"Visitando hoje esta grandiosa bibliotheca devo consignar a minha admiração não sómente pelo accumulo de tão grandes thesouros, como pela proficiencia e dedicação do seu guarda zeloso e infatigavel dr. Anțónio Constantino"

Do academico Antenor Salles de Azevedo Mello, presidente da embaixada dos alumnos da Faculdade de Direito do Recife:

"A embaixada de academicos de direito da Faculdade do Recife, ora visitando São Paulo, não expressa neste caderno de impressões a sua surpresa, porque o phenomeno da prodigiosa operosidade paulista não é regional, estende-se e empolga toda a nação. Dest'arte, o que de grande sentimos nesta bibliotheca já fazia parte do conceito que tinhamos da intelligencia paulista" 
Do professor Alfredo Russel, lente cathedratico da Faculdade de Direito da Universidade do Rio de Janeiro:

"Quem visita a Faculdade de Direito de São Paulo, onde estudaram os maiores homens do Brasil, não póde deixar de sentir-se maravilhado com as transformaçōes por que em tão pouco tempo passou. Entre o que mais merece admiração, entretanto, está a organização modelar da sua Bibliotheca, onde se encontram os aperfeiçoamentos que se têm introduzido na organização de bibliothecas. Antigo alumno da Faculdade sinto-me feliz em poder deixar assim consignada a minha impressão da visita que hoje fiz á tradicional casa de ensino".

Bahia:

Do dr. Celso Spinola, director da "Revista dos Tribunaes", da

"Visitando, de passagem por São Paulo, a esplendida Bibliotheca da sua gloriosa Faculdade, levo da mesma a melhor das impressões, pela sua moderna organização que tanto facilita a consulta daquelles que veem procural-a".

Do deputado federal e lente de direito dr. Gudesteu Pires, do Fio de Janeiro:

"A um modesto professor de direito é nimiamente grato conhecer e visitar este monumento de cultura que é a Bibliotheca da Faculdade de Direito de São Paulo".

Do professor Rodolpho Sousa Vieira, lente cathedratico da Faculdade de Direito de Goyaz:

"Grande é a emoção, que se sente ao transpôr os umbraes desta casa, por onde passaram grandes vultos da nossa historia, por isso mesmo é que nella se entra sob um respeito pouco vulgar. Sua organização, ordem e lhaneza de trato por parte de seus funccionarios tornam ainda a Faculdade mais querida dos visitantes, como acontece, agora, commigo. Sáio, assim, cheio de satisfação deste ninho de Aguias do saber, levando de tudo e de todos a mais grata recordação do que vi e ouvi".

Do desembargador Mansilio Augusto Curado Fleury, presidente da Côrte de Appellação do Estado de Goyaz: 
"Visitando a Bibliotheca desta Faculdade de Direito, admirei a ordem e esforço na conservação e melhoramento desta obra, cujos livros são restaurados. Deixo aqui a optima impressão que tive". neiro:

Do dr. Bilac Pinto, director da "Revista Forense", do Rio de Ja-

"A modelar Bibliotheca da Faculdade de Direito de São Paulo merece os louvores que podem caber num elogio sem restricções: o grande volume de obras, a organização dos ficharios, as salas de estudo, as estantes, tudo, emfim, é magnifico".

Do academico Edmundo Ferreira do Nascimento, presidente da Embaixada Cultural da Bahia:

"A Embaixada Cultural do Premio A. U. B. - da Associação Universitaria da Bahia -, visitando a Bibliotheca da Faculdade de Direito da Universidade de São Paulo, expressa, neste livro, impressão a mais brilhante não só pela organização da mesma, como ainda e principalmente pela magnifica collecção de obras raras".

Do professor José Mattos de Vasconcellos, lente da Universidade do Rio de Janeiro:

“A Bibliotheca da Faculdade de Direito de São Paulo é a expressão mais viva e eloquente da mentalidade progressista do grande povo bandeirante, que nos exalça, a nós todos brasileiros, pelas suas iniciativas culturaes em todas as fórmas e modalidades da actividade humana, nas letras, na sciencia, no commercio e na industria. Modelo de organizaçâo, esta Bibliotheca é o producto da intelligencia e da dedicação do seu bibliothecario e demais auxiliares, todos, por egual, merecedores de todos os encomios e louvores" Paulo:

Do dr. Aurelio Castello Branco, procurador da Republica em São

"Nesta rapida visita á Bibliotheca não podia deixar de exteriorizar aqui as vivas impressões sentidas pela riqueza das obras scientificas, pela magnifica cataloga- 
ção e conservação, enaltecedoras do espirito de ordem e brasilidade do grande povo bandeirante".

Do ministro Carlos Maximiliano, procurador geral da Republica:

"Amante eterno dos bons livros, manifesto, aqui, a excellente impressão causada pela rica Bibliotheca da Faculdade de Direito de São Paulo, e, sobretudo, a minha alegria de brasileiro, por verificar o interesse com que a juventude paulista prefere obras de sciencia ás de simples ficção".

Do dr. Pontes de Miranda, juiz de direito no Districto Federal:

"Vi a Bibliotheca da Faculdade de São Paulo: vae ficar á altura de São Paulo, da sua comprehensão pratica do valor do livro e das cousas espirituaes e será, de si só, um incentivo para os que, nas gerações vindouras, teem de superar a nossa geração. Um paiz sómente cresce quando a geração de hoje deixa mais do que recebeu e a geração de amanhã faz mais do que a de hoje"

Do professor Helio Gomes, lente da Universidade do Brasil, Districto Federal:

"Para quem descrê do grandioso futuro que está reservado ao Brasil, uma visita á Bibliotheca da Faculdade de Direito de São Paulo é uma obra que leva á crença nos nossos destinos. 0 homem vale, sobretudo, pelo que sabe. As bibliothecas, como esta, modelares e perfeitas, são um factor poderoso de valorização humana. Levo daqui a impressão consoladora do que o brasileiro é capaz de construir e realizar. Deixo meus applausos enthusiasticos aos que projectaram, construiram e manteem este instituto magnifico".

Do dr. Alceu Barbedo, procurador da Republica, no Rio Grande do Sul:

"Vim cumprir um dever de brasileiro, visitando a Faculdade de Direito de São Paulo, por onde passaram as gerações mais illustres da Patria. Consigno aqui, particularmente, a impressão magnifica que me deixou a Bibliotheca, organizada e dirigida pelo espirito intelligente e culto do sr. António Constantino". 
Do dr. Paulo Eliziardi, lente da Faculdade de Medicina da Universidade de Minas Geraes:

"Não poderia ser melhor a impressão causada pela minha visita á Bibliotheca da Faculdade de Direito de São Paulo, indice revelador do espirito operoso e trabalhador do povo paulista"

\section{Relação dos doadores de obras no primeiro semestre de 1937}

De 1 de janeiro a 30 de junho do corrente anno, á Bibliotheca da Faculdade de Direito fizeram doações de livros os srs. professor Francisco Morato, professor Alcantara Machado, professor Waldemar Ferreira, professor Alexandre Correia, professor M. F. Pinto Pereira, professor Spencer Vampré, dr. Bueno de Azevedo Filho, dr. Francisco A. Carvalho Franco, dr. Aureliano Leite, professor Antonio Piccarolo, desembargador Manoel Carlos de Figueiredo Ferraz, professor Candido Motta Filho, dr. Flavio Mendes, dr. Paulo Ferraz de Mesquita, dr. Amando Caiuby, dr. Paulo Vidigal de Azevedo, dr. J. Gualberto de Oliveira, dr. Frederico de Barros Brotero. dr. Henrique Machado, Nicolau Marques Schmidt, coronel Salvador de Moya, dr. Justo Seabra, Judas Isgorogota, dr. A. F. Cesarino Junior, dr. Vasco Joaquim Smith de Vasconvellos, professor Aroldo de Azevedo, dr. Jamil Miguel Nami, professor padre J. de Castro Nery, dr. Sergio Milliet da Costa e Silva, dr. Silvio Marcondes de Moura, dr. Gabriel Raja, dr. J. Rodrigues de Meréje e António Constantino, e Reitoria da Universidade, Instituto de Educação, Consulado do Japão, Commissão Central de Recenseamento, Secretaria da Agricultura, Instituto de Organização Racional do Trabalho, Consulado Geral da Italia, Liga das Senhoras Catholicas, Museu Paulista, Irmandade da Santa Casa de Misericordia, Camara Municipal da Capital, Bibliotheca da Procuradoria da Fazenda do Estado, Departamento Municipal de Cultura, Bibliotheca da Procuradoria Judicial do Estado e Consulado da Finlandia, todos de São Paulo; Officinas Graphicas do Santuario, de Apparecida do Norte; Luiz Horta Lisboa, de Campinas; Edgard de Cerqueira Falcão, de Santos; dr. Evaristo de Moraes, coronel Laurenio Lago, dr. Mario Villalva, professor José Mattos de Vasconcellos, ministro dr. José Carlos de Macedo Soares, dr. Alcides Bezerra, ministro dr. Rodrigo Octavio, dr. Renato Kiehl, coronel F. de Paula Cidade, dr. Hildebrando Accioly, M. Carlos e Jurandyr Godoy, e Embaixada Argentina, Observatorio Nacional, Instituto da Ordem dos Advogados Brasileiros, Bibliotheca da Camara dos Deputados e Directoria de Estatistica da Producção, todos do Rio de Janeiro; Secretaria do Interior do Estado de Minas Geraes, de Bello Horizonte; Selon Macedonia Soares e Gremio Mauá, de Pelotas, Rio. 
Grande do Sul; professor Rodrigues Doria e dr. Celso Spinola, da Bahia; dr. José Waldo Ribeiro Ramos, dr. Lauro Nogueira e dr. M. de Salles Andrade, de Fortaleza, Ceará; dr. Anysio Jobin, de Manáos, Amazonas; dr. J. Aureliano Correia de Araujo e dr. Annibal Bruno, de Recife, Pernambuco; dr. Adroaldo Campos e Viuva Armindo Guaraná, de Aracajú, Sergipe; dr. Rocha Filho, de Maceió, Alagoas; dr. Rodolpho Sousa Vieira e dr. Segismundo Mello, de Goyaz; professor José Farani Mansur Guerios, de Curityba, Paraná; Bibliotheca e Archivo do Estado, de Belém, Pará; dr. Edgard Queiroz do Valle e dr. Paes Barreto Filho, de Victoria, Espirito Santo; Librairie du Recueil Sirey, de Paris; professor Giorgio Del Vecchio, de Roma; professor Antonio Mendes Correia e Alberto Lemos, de Lisboa; Grupo "Amor y Vida", de Barcelona, Espanha; Direción del Registro de Estado Civil, de Montevideo; Elizabeth Sprague Coolidge Foundation, de Washington; Rodolfo A. Vaccarezza e dr. Eugenio Osvaldo Cardini, de Buenos Aires; Association Suisse des Électreciens, de Zurich; The Hispanic Society of America e Rensselaer Polytechnic Institute, de New York; e Departamento Autónomo de Publicidad y Propaganda, de Mexico. 